\title{
Molecular Mechanisms and Epidemiology of Carbapenem-Resistant Enterobacter cloacae Complex Isolated from Chinese Patients During 2004-2018
}

\author{
Shixing Liu' \\ Na Huang' \\ Cui Zhou' \\ Yishuai Lin ${ }^{2}$ \\ Ying Zhang ${ }^{2}$ \\ Lingbo Wang' \\ Xiangkuo Zheng' \\ Tieli Zhou' \\ Zhongyong Wang' \\ 'Department of Clinical Laboratory, The \\ First Affiliated Hospital of Wenzhou \\ Medical University, Wenzhou, Zhejiang \\ Province, People's Republic of China; \\ ${ }^{2}$ School of Laboratory Medicine and Life \\ Science, Wenzhou Medical University, \\ Wenzhou, Zhejiang Province, People's \\ Republic of China
}

Correspondence: Zhongyong Wang; Tieli Zhou

Department of Clinical Laboratory, The First Affiliated Hospital of Wenzhou Medical University, Wenzhou, Zhejiang

Province, People's Republic of China

Tel +86-577-5557-9725;

$+86-577-8668-9885$

Email wangforever2000@163.com; wyztli@I63.com
Background: The emergence and spread of carbapenem-resistant Enterobacter cloacae complex (ECC) have posed a serious threat to human health worldwide. This study aimed to investigate the molecular mechanism of carbapenem resistance and its prevalence among ECC in China.

Methods: A total of 1314 ECC clinical isolates were collected from the First Affiliated Hospital of Wenzhou Medical University from 2004 to 2018. Sensitivity to antibiotics was determined using the agar dilution method. The production of carbapenemases and the prevalence of resistance-associated genes were investigated using PCR. The expression of outer membrane porin (OMP) genes (ompC/ompF) and cephalosporinase gene ampC was analyzed by quantitative real-time PCR. The effect of efflux pump mechanism on carbapenem resistance was tested. ECC was typed by multilocus sequence typing (MLST).

Results: In this study, 113 carbapenem-nonsusceptible ECC strains were identified. The prevalence rates of carbapenemase genes $b l a_{\mathrm{KPC}-2}$ and $b l a_{\mathrm{NDM}}$ were $12.4 \%(14 / 113)$ and $17.7 \%(20 / 113)$, and that of the extended-spectrum $\beta$-lactamase (ESBL) genes bla $a_{\mathrm{CTX}-\mathrm{M}}$, $b l a_{\mathrm{TEM}}$, and $b l a_{\mathrm{SHV}}$ were $28.3 \%(32 / 113), 27.4 \%$ (31/113), and $14.2 \%$ (16/113), respectively. Among 67 carbapenem-nonsusceptible ECC isolates producing non-carbapenemase, low expression of ompC/ompF and overexpression of ampC were found in 46 and 40 strains, respectively. In addition, the carbapenem resistance was related to the overexpression of the efflux pump in the study. Finally, the 113 carbapenem-nonsusceptible ECC strains were categorized into 39 different sequence types using MLST.

Conclusion: Carbapenem-nonsusceptible ECC strains producing non-carbapenemase were predominant. The low expression of OMP with the overexpression of cephalosporinase or production of ESBLs and overexpression of efflux pump might contribute to the resistance to carbapenem for carbapenem-nonsusceptible ECC strains producing non-carbapenemase. The $b l a_{\mathrm{NDM}}$ and $b l a_{\mathrm{KPC}}$ comprised the principal resistance mechanism of carbapenemase-producing ECC in the hospital, causing a threat to public health. Therefore, monitoring programs to prevent the emergence and further spread of antibiotic resistance are urgently needed.

Keywords: carbapenemase, carbapenem-resistant mechanism, Enterobacter cloacae complex, epidemiology, non-carbapenemase

\section{Introduction}

The Enterobacter cloacae complex (ECC) is one of the common microorganisms isolated in clinical specimens causing all kinds of infections, like pneumonia, urinary tract infections, and sepsis, in the last few decades. ${ }^{1,2}$ Multidrug-resistant (MDR) ECC isolates have emerged and spread worldwide with the widespread use of antibiotics. ${ }^{3}$ With the increase in resistance rates to aminoglycosides, 
fluoroquinolones, and third-generation cephalosporins, carbapenems, as the last-resort antibiotic, have gradually been used for treating MDR ECC infections. ${ }^{1,4,5}$ However, the increasing resistance rates of carbapenems have gained special clinical attention. ${ }^{6-8}$

The mechanisms of carbapenem resistance in ECC are realized by either the acquisition of plasmid-encoded carbapenemase genes and the overexpression of efflux pumps, or, more commonly, the constitutive overexpression of AmpC or production of extended-spectrum $\beta$-lactamase (ESBL) combined with disrupted membrane permeability (the decrease in or loss of the outer membrane protein). ${ }^{3,9} \mathrm{ECC}$ is inherently resistant to first- and second-generation cephalosporins because the overexpression of an inducible AmpC $\beta$ lactamase is encoded by the chromosome gene $\operatorname{amp} C{ }^{3,10}$ Moreover, the acquisition of plasmid-mediated ESBL genes, such as $b l a_{\mathrm{TEM}}, b l a_{\mathrm{SHV}}, b l a_{\mathrm{CTX}-\mathrm{M}}$, and so forth, makes ECC resistant to most $\beta$-lactam drugs, thus increasing the difficulty in clinical treatment. ${ }^{11-15}$ Two major categories of carbapenem enzymes, carbapenem-hydrolyzing serine $\beta$-lactamases and metallo- $\beta$-lactamases, such as KPC, NmcA, IMI, FRI, GES, OXA, VIM, IMP, and NDM, have been identified in carbapenem-resistant Enterobacteriaceae. ${ }^{3}$ The most common description of KPC and NDM-1 was in ECC isolates. ${ }^{16}$

Considering the increasing prevalence of carbapenemnonsusceptible ECC isolates worldwide, longitudinal epidemiological surveillance and resistance mechanism study on the carbapenem-nonsusceptible ECC should be performed to control and prevent the distribution and spread of resistance, which is key to clinical significance in guiding antimicrobial therapy. However, relevant data on the long-term evolution of carbapenem-nonsusceptible ECC are lacking in China. In this study, the epidemiology prevalence and the molecular mechanisms of 113 ECC clinical isolates were characterized for carbapenem resistance during large-scale surveillance in the southeast of China. This study was novel in reporting ECC nonsusceptible to carbapenem antibiotics on a large scale in China.

\section{Materials and Methods}

\section{Bacterial Isolates}

A total of 1314 ECC clinical isolates were collected from the First Affiliated Hospital of Wenzhou Medical University (Wenzhou, China) from 2004 to 2018. Antimicrobial susceptibility testing and identification of all isolates were performed using a VITEK ${ }^{\circledR} 2$ system (bioMérieux, Marcyl'Étoile, France). The isolates were stored in 30\% glycerol at $-80^{\circ} \mathrm{C}$ prior to further analysis. All investigation protocols in this study were approved by the ethics committee of the First Affiliated Hospital of Wenzhou Medical University. Informed consent was waived because this study with observational nature focused mainly on bacteria and involved no interventions to patients.

\section{Minimum Inhibitory Concentration Determination}

According to the latest guidelines recommended by the Institute of Clinical and Laboratory Standards (CLSI, 2020), the minimum inhibitory concentrations (MICs) of 15 antimicrobial agents, including aztreonam (ATM), ciprofloxacin (CIP), levofloxacin (LVX), ceftriaxone (CRO), cefepime (FEP), ceftazidime (CAZ), meropenem (MEM), imipenem (IPM), ertapenem (ETP), gentamicin (GEN), tobramycin (TOB), amikacin (AMK), trimethoprim-sulfamethoxazole (SXT), nitrofurantoin (NIT), and colistin (COL), were determined by the agar dilution method. Briefly, the bacteria were suspended in saline to one-tenth the turbidity of the $0.5 \mathrm{McF}$ arland standard. The bacterial suspension was inoculated on the MuellerHinton (MH) agar plate containing different drug concentrations using the nail plate. The results were quantified by observing bacterial growth after incubation at $37^{\circ} \mathrm{C}$ for 16-20 h. ${ }^{17}$ The MIC determination of colistin was explained by the recommendation of the European Committee on Antimicrobial Susceptibility Testing. Escherichia coli ATCC 25922 was used as the quality control strain for antimicrobial susceptibility testing. All experiments were performed in triplicate.

\section{Detection of Extended-Spectrum Beta-Lactamases}

The American Clinical Laboratory Standardization Institute (CLSI) recommended ESBL confirmation test was performed. ${ }^{1}$ Briefly, a lawn of test bacteria suspension equivalent to $0.5 \mathrm{McF}$ arland turbidity standard solution was swabbed on the surfaces of MHA plates, and then ceftazidime $(30 \mu \mathrm{g})$ and cefotaxime $(30 \mu \mathrm{g})$ disks (Kanvax, China) with and without clavulanic acid $(10 \mu \mathrm{g})$ were seeded within $15 \mathrm{~min}$. All plates were then incubated aerobically at $37^{\circ} \mathrm{C}$ for $18 \mathrm{~h}$. An isolate was phenotypically confirmed as an ESBL producer when a zone diameter difference of $\geq 5 \mathrm{~mm}$ was observed between both antibiotic disks with clavulanic acid and a similar agent without clavulanic acid. ${ }^{18}$ The E. coli ATCC 25922 strain was 
used as the negative control, and the Klebsiella pneumoniae ATCC 700603 strain was used as the positive control.

\section{Detection of Antibiotic Resistance Determinants}

The beta-lactamase genes, including carbapenemase genes (bla $a_{\mathrm{KPC}}, \quad b l a_{\mathrm{IMP}}, \quad b l a_{\mathrm{NDM}}, \quad b l a_{\mathrm{SPM}}, \quad b l a_{\mathrm{IMI}}, \quad b l a_{\mathrm{VIM}}$, bla $a_{\mathrm{OXA}-23}, b l a_{\mathrm{OXA}-24}, \quad b l a_{\mathrm{OXA}-48}, \quad b l a_{\mathrm{OXA}-58}, b l a_{\mathrm{Nmc-A}}$, $b l a_{\mathrm{FRI}-1}, b l a_{\mathrm{SME}}, b l a_{\mathrm{GIM}}, b l a_{\mathrm{BIC}}, b l a_{\mathrm{DIM}}, b l a_{\mathrm{AIM}}, b l a_{\mathrm{GES}}$, and $\left.b l a_{\mathrm{SIM}}\right)$ and extended-spectrum $\beta$-lactamase genes (bla $a_{\mathrm{TEM}}, b l a_{\mathrm{SHV}}, b l a_{\mathrm{CTX}-\mathrm{M}-1}, b l a_{\mathrm{CTX}-\mathrm{M}-9}$, and $\left.b l a_{\mathrm{CTX}-\mathrm{M}-14}\right)$ , were identified by polymerase chain reaction (PCR). The primers of all genes are summarized in Table S1. The positive products of PCR amplification were sequenced by Shanghai Genomics Institute Technology Co. Ltd. (Shanghai, China). All sequencing results of the products were analyzed using BLAST searches against the NCBI database (www.ncbi.nlm.nih.gov/BLAST).

\section{Efflux Pump Inhibition Assay}

The efflux pump activity of carbapenem-insensitive ECC strains was determined using the efflux pump inhibitor carbonyl cyanide 3-chlorophenylhydrazone (CCCP) (Sigma, MO, USA). The MICs of ertapenem in the presence or absence of $8 \mu \mathrm{g} / \mathrm{mL}$ CCCP, which did not inhibit the growth of ECC bacteria, were determined by the agar dilution method. In the presence of CCCP, a reduction in carbapenem $\mathrm{MIC} \geq 4$ times was defined as the positive phenotype of the efflux pump downregulation.

\section{Quantitative Real-Time PCR for Cephalosporinase AmpC and Outer Membrane Protein}

The total RNA of 67 noncarbapenemase-producing ECC isolates was extracted. Then, $500 \mathrm{ng}$ RNA was mixed with the reverse transcription system, and $10 \mu \mathrm{L}$ of cDNA was obtained using a PrimeScript ${ }^{\mathrm{TM}}$ RT Kit (TaKaRa, Japan). Using a CFX96 touch real-time PCR system, qPCR (Bio-Rad, CA, USA) was performed. Then, 100 ng cDNA, TB Green Premix Ex Taq II (Tli RNaseH Plus) $(2 \times)$ (TaKaRa), and specific primers (ompc f: 5'-GCGACCAGACCTACATGCGT-3', r: 5'TTCGTTCTCACCAGAGTTACCCT-3', ompF f: 5'-TCCC TGCCCTGCTGGTAG-3'， r: 5'-TAAGTGTTGTCGCCAT CGTTG-3', ampC f: 5'-GCATGGCGGTGGCCGTTAT-' r: 5'CTGCTTGCCCGTCAGCTGT-3') were added to each sample. The cycling conditions were as follows: $95^{\circ} \mathrm{C}$ for $30 \mathrm{~s}$, followed by 40 cycles of $95^{\circ} \mathrm{C}$ for $5 \mathrm{~s}$ and $60^{\circ} \mathrm{C}$ for $30 \mathrm{~s}$. The expression levels of outer membrane genes ompC and $o m p F$ and cephalosporinase gene $a m p C$ were detected by RT-qPCR; the $r p o B$ gene was used as the internal gene. Compared with carbapenem-sensitive Enterobacter cloacae ATCC700323, the target genes were quantified using the comparative threshold cycle $2^{-\Delta \Delta \mathrm{Ct}}$ method. All experiments were repeated three times independently and averaged in the calculation of relative expression levels.

\section{Multilocus Sequence Typing}

The carbapenem-nonsusceptible ECC isolates were analyzed by amplifying seven housekeeping genes (dnaA, fus $A, \operatorname{gyr} B$, leuS, pyrG, rplB, and $r p o B$ ). Sequence types (STs) were assigned by querying against the database available at the Institut Pasteur's Enterobacter cloacae MLST website (https://pubmlst.org/bigsdb? db=pubmlst ecloacae seqdef). Following the genetic similarity diagram using the goeBURST program, the clonal complexes were analyzed to identify the molecular epidemiological relationships.

\section{Results}

\section{Bacterial Isolates and Antimicrobial Susceptibility Test}

A total of $113 / 1314(8.6 \%)$ carbapenem-nonsusceptible ECC isolates were determined with imipenem, meropenem, and ertapenem; 99 (7.5\%) carbapenem-resistant strains were determined. The carbapenem-resistant ECC isolate was first detected in the hospital in 2010; the resistance rate increased from $2.5 \%$ in 2010 to $11.9 \%$ in 2018 (Table 1). The results of the antimicrobial susceptibility test of all 113 carbapenem-nonsusceptible isolates are listed in Table 2, which suggested higher resistance to fluoroquinolones, cephalosporins, and monobactams. Further, 63 (55.8\%) and 45 (39.8\%) ECC isolates were resistant to ciprofloxacin and levofloxacin, respectively; 30 $(26.5 \%)$ and $35(31.0 \%)$ isolates were resistant to gentamicin and tobramycin, respectively. Of note, 112 (99\%) ECC strains were resistant to ceftriaxone. Nevertheless, 7 $(6.2 \%)$ and $20(17.7 \%)$ isolates were resistant to amikacin and colistin, respectively.

\section{Frequency of $\beta$-Lactamase Genes}

A total of 46 carbapenem-nonsusceptible ECC isolates carried carbapenemase genes (Figure 1). The prevalence rate of bla $_{\mathrm{KPC}-2}, b l a_{\mathrm{NDM}}$, bla $_{\mathrm{IMP}}$, and bla $_{\mathrm{OXA}-23}$ in carbapenem-nonsusceptible strains was 12.4\% (14/113), 17.7\% 
Table I Carbapenem Susceptibility of ECC Clinical Isolates

\begin{tabular}{|l|c|c|c|c|c|c|}
\hline $\begin{array}{l}\text { Time of } \\
\text { Isolation }\end{array}$ & $\begin{array}{c}\text { No. of } \\
\text { Isolates }\end{array}$ & $\begin{array}{c}\text { CNS (R } \\
\text { I) (n) }\end{array}$ & $\begin{array}{c}\text { NS } \\
\text { (\%) }\end{array}$ & $\mathbf{R}$ (\%) & I (\%) & S (\%) \\
\hline 2004 & 32 & 0 & 0 & 0 & 0 & 100 \\
\hline 2005 & 46 & 0 & 0 & 0 & 0 & 100 \\
\hline 2006 & 47 & 0 & 0 & 0 & 0 & 100 \\
\hline 2007 & 47 & 0 & 0 & 0 & 0 & 100 \\
\hline 2008 & 60 & 0 & 0 & 0 & 0 & 100 \\
\hline 2009 & 41 & 0 & 0 & 0 & 0 & 100 \\
\hline 2010 & 40 & $I(I+0)$ & 2.5 & 2.5 & 0 & 97.5 \\
\hline 2011 & 34 & $5(4+1)$ & 14.7 & 11.8 & 2.9 & 85.3 \\
\hline 2012 & 82 & $6(5+1)$ & 7.3 & 6.1 & 1.2 & 92.7 \\
\hline 2013 & 36 & $I(I+0)$ & 2.8 & 2.8 & 0 & 97.2 \\
\hline 2014 & 36 & $4(4+0)$ & 11.1 & 11.1 & 0 & 88.9 \\
\hline 2015 & 177 & $20(19+1)$ & 11.3 & 10.7 & 0.6 & 88.7 \\
\hline 2016 & 214 & $31(25+6)$ & 14.5 & 11.7 & 2.8 & 85.5 \\
\hline 2017 & 221 & $20(16+4)$ & 9.0 & 7.2 & 1.8 & 91.0 \\
\hline 2018 & 201 & $25(24+1)$ & 12.4 & 11.9 & 0.5 & 87.6 \\
\hline Total & 1314 & $113(99+14)$ & 8.6 & 7.5 & 1.1 & 91.4 \\
\hline
\end{tabular}

Abbreviations: ECC, Enterobacter cloacae complex; No., number; CNS, carbapenem-nonsusceptible strains; $S$, sensitivity; I, intermediate; R, resistance; NS; R and I.

(20/113), 8.0\% (9/113), and 3.5\% (4/113), respectively, including one isolate carrying $b l a_{\mathrm{KPC}-2}$ and $b l a_{\mathrm{NDM}-1}$ specially (Figure 1), while bla $a_{\mathrm{Nmc}-\mathrm{A}}, b l a_{\mathrm{BIC}}, b l a_{\mathrm{GES}}, b l a_{\mathrm{AIM}}$, $b l a_{\mathrm{GIM}}, b l a_{\mathrm{DIM}}, b l a_{\mathrm{SIM}}, b l a_{\mathrm{OXA}-24}, b l a_{\mathrm{OXA}-48}, b l a_{\mathrm{FRI}-1}$, $b l a_{\mathrm{OXA}-58}, b l a_{\mathrm{SME}}$, and $b l a_{\mathrm{AIM}}$ were not detected in all isolates. Moreover, $49 \mathrm{ECC}$ strains were positive for the ESBL phenotypic test in 113 strains, and the ESBLs genes bla $_{\mathrm{TEM}}(27.4 \%, 31 / 113)$, bla CTХ-М-14 $(19.5 \%, 22 / 113)$, $b_{\text {CTX-M-1 }}(15.9 \%, 18 / 113)$, bla $a_{\text {СТХ-М-9 }}(14.2 \%, 16 / 113)$, and bla $_{\mathrm{SHV}}(14.2 \%, 16 / 113)$ among analyzed strains were also determined. In general, the harboring of carbapenemase genes and ESBLs accounted for 40.7\% (46/113) and $58.4 \%$ (66/113), respectively.

\section{Overexpression of the Efflux Pump}

The efflux pump inhibition test was performed to explore the effect of the efflux pump on carbapenem resistance. The results showed that 36 strains had $\mathrm{a} \geq 4$ times reduction in ertapenem MICs in the presence of $8 \mu \mathrm{g} / \mathrm{mL}$ CCCP, suggesting that the efflux pump had a significant effect on carbapenem resistance.

\section{Outer Membrane Protein Gene Expressions}

This study investigated the relationship between resistance to carbapenems and the expression level of outer membrane genes ompC and ompF and cephalosporinase gene ampC. Further, 67 ECC noncarbapenemase-producing isolates and Enterobacter cloacae ATCC700323 as the control strain were used. The results of RT-qPCR showed that the expression level of ompC in 29 ECC isolates decreased compared with that in ATCC 700323, and the decreased expression of ompF was found in 42 ECC strains. In addition, 24 ECC isolates had low expression of both отрC and $о т р F$. The overexpression of cephalosporinase gene $a m p C$ was found in 40 ECC strains, with the highest

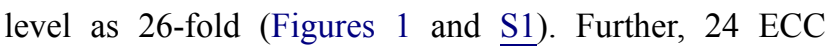
isolates had the overexpression of cephalosporinase gene ampC and low expression of $о т p C$ or/and $о т p F$. ESBLs and low-level expression of $о т p C$ or/and $о m p F$ were detected in 33 ECC isolates (Figure 1).

\section{Multilocus Sequence Typing Analysis}

The 113 carbapenem-nonsusceptible ECC isolates were assigned to 39 different STs, including the most prevalent ST190 (14.2\%, 16/113), followed by ST114 (4.4\%, 5/113), ST93 (4.4\%, 5/113), ST97 (3.5\%, 4/113), ST78 (3.5\%, 4/ 113), ST32 (2.7\%, 3/113), ST46 (1.8\%, 2/113), ST120 (1.8\%, 2/113), ST523 (1.8\%, 2/113), ST557 (1.8\%, 2/ $113), \operatorname{ST} 1411(1.8 \%, 2 / 113)$, and ST1439 (1.8\%, 2/113); the remaining STs contained 1 strain for each, except 34 novel STs (marked as NEW in Figure 1, currently not registered in the MLST database) as detected by MLST analysis (Figure 1). Also, 39 STs were analyzed for the molecular epidemiological relationships using goeBURST. The result showed that all 39 STs belonged to singletons with no CCs (Figure 2). In addition, of 16 ST190 strains, 4 isolates produced KPC-2, 1 produced NDM-1, 1 produced OXA-23, 1 produced IMP, and other produced ESBLs. All ST114 isolates produced NDM, including three (75\%) strains with $b l a_{\mathrm{NDM}-1}$, and two produced NDM-5. All ST97 strains produced carbapenemase; three strains carried $b l a_{\mathrm{NDM}-1}$ and one $b l a_{\mathrm{IMP}}$.

\section{Discussion}

Carbapenem is widely used in the treatment and management of MDR Gram-negative bacterial infections in the clinical environment due to its broad-spectrum antibacterial activity. ${ }^{19}$ However, some surveillance programs 
Table 2 Minimum Inhibitory Concentrations (MICs) of I I 3 Carbapenem-Nonsusceptible ECC Isolates

\begin{tabular}{|c|c|c|c|c|c|c|c|c|c|c|c|c|c|c|c|}
\hline \multirow[t]{2}{*}{ Isolates } & \multicolumn{15}{|c|}{ MIC $(\mu \mathrm{g} / \mathrm{mL})$} \\
\hline & ATM & CRO & CAZ & FEP & ETP & IPM & MEM & CIP & LVX & GEN & тOB & AMK & SXT & NIT & COL \\
\hline Y509 & $\leq 1$ & $\geq 64$ & $\geq 64$ & $\geq 64$ & 16 & 2 & 4 & I & 1 & $\geq 16$ & $\geq 16$ & 8 & $\geq 320$ & I & 0.25 \\
\hline Y54। & 64 & $\geq 32$ & $\geq 128$ & 32 & 2 & 0.5 & $\leq 0.125$ & 0.06 & 0.06 & 4 & 2 & 4 & $\geq 320$ & 64 & 8 \\
\hline Y542 & 32 & $\geq 32$ & $\geq 128$ & 4 & 2 & 0.5 & 0.25 & 0.25 & 0.06 & 2 & 0.5 & 2 & 20 & 64 & 0.125 \\
\hline Y569 & 32 & $\geq 32$ & $\geq 128$ & 4 & I & $\leq 0.125$ & $\leq 0.125$ & 0.06 & 0.06 & 2 & 0.5 & 2 & $\leq 5$ & 32 & 0.125 \\
\hline Y572 & 32 & $\geq 32$ & $\geq 128$ & I & 2 & 0.5 & 0.5 & 0.06 & 0.06 & 2 & 2 & 8 & 10 & 32 & 0.25 \\
\hline Y573 & $\geq 128$ & $\geq 32$ & $\geq 128$ & 8 & 2 & 0.5 & 0.25 & $\geq 8$ & $\geq 16$ & 64 & 16 & $\geq 512$ & $\geq 320$ & 128 & 0.25 \\
\hline CG35 & 0.5 & $\geq 32$ & $\geq 128$ & 16 & 2 & 8 & 4 & 0.25 & 0.5 & 8 & 8 & 4 & $\geq 320$ & 32 & 0.125 \\
\hline CG38 & I & $\geq 32$ & $\geq 128$ & 32 & 64 & 64 & 64 & 0.5 & I & 16 & 8 & 4 & $\geq 320$ & 32 & 0.25 \\
\hline CG64 & 32 & $\geq 32$ & $\geq 128$ & 32 & 8 & 4 & 4 & $\geq 8$ & $\geq 16$ & 4 & 16 & 4 & $\geq 320$ & 32 & 0.25 \\
\hline CG80 & 64 & $\geq 32$ & $\geq 128$ & 8 & I & I & 0.25 & 1 & I & 4 & 4 & I & $\geq 320$ & 64 & 0.125 \\
\hline CG85 & 0.5 & 4 & 16 & 0.06 & 4 & 2 & 0.25 & 0.5 & 0.06 & 4 & 4 & 8 & $\leq 5$ & 64 & 0.125 \\
\hline CG98 & 64 & 16 & 8 & 8 & 128 & 8 & 16 & $\geq 8$ & $\geq 16$ & 32 & 4 & 4 & $\geq 320$ & $\geq 1024$ & 0.5 \\
\hline CGI75 & 4 & $\geq 32$ & $\geq 128$ & $\geq 128$ & $>128$ & 64 & 64 & I & I & 8 & 8 & 4 & $\geq 320$ & 128 & $>64$ \\
\hline CG37I & $\geq 128$ & $\geq 32$ & $\geq 128$ & 8 & 8 & 2 & 4 & 0.5 & 0.25 & $\geq 128$ & 64 & 16 & $\geq 320$ & 32 & 0.25 \\
\hline CG380 & 64 & $\geq 32$ & $\geq 128$ & 4 & 2 & 0.25 & $\leq 0.125$ & 0.25 & 0.03 & 1 & 0.5 & 2 & 40 & 64 & 0.125 \\
\hline CG389 & $\geq 128$ & $\geq 32$ & 16 & 64 & 2 & I & 0.25 & $\geq 8$ & $\geq 16$ & $\geq 128$ & $\geq 128$ & $\geq 512$ & $\geq 320$ & 256 & 0.25 \\
\hline CG4I7 & 32 & $\geq 32$ & 64 & 2 & 4 & 0.5 & 0.25 & 4 & 8 & I & 0.5 & 4 & 80 & 128 & I \\
\hline CG586 & $\leq 1$ & $\geq 64$ & $\geq 64$ & $\geq 64$ & 16 & 2 & 4 & I & 1 & $\geq 16$ & $\geq 16$ & 8 & $\geq 320$ & 64 & 0.5 \\
\hline CG604 & $\geq 64$ & 32 & $\geq 64$ & 4 & 2 & 0.5 & 0.25 & 2 & 4 & 8 & $\geq 16$ & 16 & $\geq 320$ & 128 & 0.5 \\
\hline CG648 & 16 & 16 & 4 & 4 & 4 & 4 & 4 & $\leq 0.25$ & $\leq 0.25$ & $\leq 1$ & $\leq 0.25$ & $\leq 0.25$ & $\leq 5$ & 32 & $>64$ \\
\hline CG652 & 32 & $\geq 32$ & $\geq 128$ & 2 & 2 & 0.25 & $\leq 0.125$ & 0.25 & 0.5 & 2 & 1 & 4 & $\leq 5$ & 64 & 0.25 \\
\hline CG662 & 8 & $\geq 32$ & $\geq 128$ & 8 & 2 & 4 & I & 0.25 & 0.25 & 64 & 4 & 4 & $\geq 320$ & 16 & 0.5 \\
\hline CG666 & 32 & $\geq 64$ & $\geq 64$ & 4 & 2 & 0.25 & 0.25 & $\leq 0.25$ & $\leq 0.25$ & $\leq 1$ & $\leq 1$ & $\leq 2$ & $\leq 20$ & 32 & 0.25 \\
\hline CG698 & $\leq 1$ & $\geq 64$ & $\geq 64$ & 8 & 2 & I & 0.5 & $\leq 0.25$ & I & 8 & 4 & $\leq 2$ & $\geq 320$ & 64 & $>64$ \\
\hline CG709 & 1 & 16 & $\geq 128$ & 2 & 2 & 0.5 & $\leq 0.125$ & 0.5 & 0.25 & 32 & 16 & 2 & $\geq 320$ & 32 & 0.5 \\
\hline CG72I & $\geq 64$ & $\geq 64$ & $\geq 64$ & $\geq 64$ & 2 & I & 0.25 & $\geq 4$ & $\geq 8$ & $\leq 1$ & 8 & $\leq 2$ & $\leq 20$ & 256 & 0.5 \\
\hline CG727 & $\geq 64$ & $\geq 64$ & $\geq 64$ & $\leq 1$ & 1 & 0.5 & 0.25 & $\leq 0.25$ & $\leq 0.25$ & $\leq 1$ & $\leq 1$ & $\leq 2$ & $\leq 20$ & 64 & $>64$ \\
\hline CG737 & $\leq 1$ & 4 & 2 & $\leq 1$ & 4 & 0.5 & $\leq 0.125$ & $\geq 4$ & $\geq 8$ & $\leq 1$ & 8 & $\leq 2$ & $\geq 320$ & 64 & $>64$ \\
\hline CG74I & $\leq 1$ & 4 & 2 & $\leq 1$ & 4 & 0.25 & $\leq 0.125$ & $\geq 4$ & $\geq 8$ & $\leq 1$ & 8 & $\leq 2$ & $\geq 320$ & 64 & 64 \\
\hline CG745 & $\geq 128$ & $\geq 32$ & $\geq 128$ & 16 & 4 & 0.5 & I & $\geq 8$ & $\geq 16$ & I & 1 & 2 & $\geq 320$ & 64 & 0.5 \\
\hline CG749 & $\leq 1$ & $\geq 64$ & $\geq 64$ & $\geq 64$ & 128 & 8 & 16 & $\geq 4$ & $\geq 8$ & $\geq 16$ & $\geq 16$ & 4 & $\geq 320$ & 64 & 0.5 \\
\hline CG780 & 8 & $\geq 32$ & 64 & 0.5 & 4 & 0.5 & $\leq 0.125$ & $\geq 8$ & $\geq 16$ & 1 & 1 & 2 & $\geq 320$ & 128 & 0.5 \\
\hline
\end{tabular}


Table 2 (Continued).

\begin{tabular}{|c|c|c|c|c|c|c|c|c|c|c|c|c|c|c|c|}
\hline \multirow[t]{2}{*}{ Isolates } & \multicolumn{15}{|c|}{ MIC $(\mu \mathrm{g} / \mathrm{mL})$} \\
\hline & ATM & CRO & CAZ & FEP & ETP & IPM & MEM & CIP & LVX & GEN & TOB & AMK & SXT & NIT & COL \\
\hline CG804 & $\geq 128$ & $\geq 32$ & $\geq 128$ & 64 & 32 & 4 & 4 & $\geq 8$ & $\geq 16$ & 2 & I & 4 & $\geq 320$ & 32 & 0.5 \\
\hline CG8I5 & $\geq 64$ & $\geq 64$ & 16 & 8 & 16 & 16 & 4 & $\leq 0.25$ & $\leq 0.25$ & $\leq 1$ & $\leq 1$ & $\leq 2$ & $\leq 20$ & 64 & 0.5 \\
\hline CG8I7 & $\geq 64$ & 32 & 16 & 2 & 8 & 4 & I & $\leq 0.25$ & $\leq 0.25$ & $\leq 1$ & $\leq 1$ & $\leq 2$ & $\leq 20$ & 32 & 0.5 \\
\hline CG70I & 2 & $\geq 64$ & 2 & 32 & 2 & 2 & 0.25 & $\leq 0.25$ & 1 & $\geq 16$ & 8 & $\leq 2$ & $\geq 320$ & 32 & $>64$ \\
\hline CG718 & $\leq 1$ & $\leq 1$ & $\leq 1$ & $\leq 1$ & 0.25 & 4 & $\leq 0.125$ & 0.5 & 1 & $\leq 1$ & $\leq 1$ & $\leq 2$ & $\leq 20$ & 64 & 0.5 \\
\hline CG824 & $\geq 64$ & $\geq 64$ & $\geq 64$ & 2 & I & 0.25 & $\leq 0.125$ & $\leq 0.25$ & $\leq 0.25$ & $\leq 1$ & $\leq 1$ & $\leq 2$ & $\leq 20$ & 64 & 0.5 \\
\hline CG848 & $\geq 64$ & $\geq 64$ & $\geq 64$ & 16 & 4 & 0.5 & 0,25 & $\geq 4$ & 4 & $\leq 1$ & $\leq 1$ & $\leq 2$ & $\leq 20$ & 64 & 0.5 \\
\hline CG864 & $\geq 64$ & $\geq 64$ & $\geq 64$ & 4 & 8 & 0.5 & $\leq 0.125$ & $\leq 0.25$ & $\leq 0.25$ & $\leq 1$ & $\leq 1$ & $\leq 2$ & $\leq 20$ & 1 & $>64$ \\
\hline CG87I & $\geq 64$ & $\geq 64$ & $\geq 64$ & 2 & 2 & 0.5 & 0.125 & $\leq 0.25$ & 0.5 & $\leq 1$ & $\leq 1$ & $\leq 2$ & $\leq 20$ & I & 0.5 \\
\hline CG884 & $\geq 64$ & $\geq 64$ & $\geq 64$ & $\leq 1$ & 2 & 0.5 & $\leq 0.125$ & $\leq 0.25$ & I & $\leq 1$ & $\leq 1$ & $\leq 2$ & $\leq 20$ & I & 32 \\
\hline CG90I & 64 & $\geq 32$ & $\geq 128$ & 64 & 16 & 2 & 2 & $\geq 8$ & 8 & 64 & $\geq 128$ & $\geq 512$ & $\geq 320$ & 256 & 0.5 \\
\hline CG9II & $\geq 64$ & $\geq 64$ & 16 & 16 & 16 & 8 & 4 & 0.5 & I & $\geq 16$ & $\geq 16$ & 16 & $\geq 320$ & 64 & 0.5 \\
\hline CG914 & $\geq 64$ & $\geq 64$ & 16 & 16 & 32 & 4 & 4 & 0.5 & I & $\geq 16$ & $\geq 16$ & 16 & $\geq 320$ & 64 & 0.5 \\
\hline CG916 & $\geq 64$ & $\geq 64$ & $\geq 64$ & $\geq 64$ & 128 & 16 & 16 & 0.5 & I & $\geq 16$ & $\geq 16$ & $\leq 2$ & $\geq 320$ & 64 & 0.5 \\
\hline CG934 & $\geq 64$ & $\geq 64$ & $\geq 64$ & $\leq 1$ & 2 & 0.25 & $\leq 0.125$ & $\leq 0.25$ & $\leq 0.25$ & $\leq 1$ & $\leq 1$ & $\leq 2$ & $\leq 20$ & $\leq 16$ & $>64$ \\
\hline CG937 & 16 & $\geq 64$ & $\geq 64$ & $\leq 1$ & 2 & 0.5 & $\leq 0.125$ & $\geq 4$ & $\geq 8$ & $\geq 16$ & $\geq 16$ & $\geq 64$ & $\geq 320$ & 256 & 0.5 \\
\hline CG939 & $\geq 64$ & $\geq 64$ & $\geq 64$ & 4 & I & 0.5 & $\leq 0.125$ & I & 1 & 8 & $\geq 16$ & 16 & $\geq 320$ & 64 & 0.5 \\
\hline CG945 & $\geq 64$ & $\geq 64$ & $\geq 64$ & 16 & 4 & 0.5 & $\leq 0.125$ & I & 2 & 8 & $\geq 16$ & 16 & $\geq 320$ & 64 & 0.5 \\
\hline CG947 & $\geq 64$ & $\geq 64$ & $\geq 64$ & 16 & I & 0.5 & 0.25 & I & 2 & 8 & $\geq 16$ & 16 & $\geq 320$ & 64 & 0.5 \\
\hline CG950 & $\geq 64$ & $\geq 64$ & $\geq 64$ & 4 & 2 & 0.5 & $\leq 0.125$ & I & 2 & 8 & $\geq 16$ & 16 & $\geq 320$ & 64 & 0.5 \\
\hline CG952 & $\geq 64$ & $\geq 64$ & $\geq 64$ & 4 & 4 & 0.5 & 0.5 & I & 2 & 8 & $\geq 16$ & 16 & $\geq 320$ & 64 & 0.5 \\
\hline CG983 & 16 & $\geq 64$ & $\geq 64$ & $\geq 64$ & 128 & 8 & 16 & $\geq 4$ & $\geq 8$ & $\geq 16$ & $\geq 16$ & $\geq 64$ & $\leq 20$ & 256 & 0.5 \\
\hline CG996 & 4 & $\geq 64$ & $\geq 64$ & 16 & 8 & 2 & I & $\leq 0.25$ & $\leq 0.25$ & $\geq 16$ & $\geq 16$ & $\leq 2$ & $\leq 20$ & 64 & 0.5 \\
\hline CGI005 & $\geq 64$ & $\geq 64$ & $\geq 64$ & $\geq 64$ & $>256$ & 64 & 128 & $\geq 4$ & 4 & $\geq 16$ & $\geq 16$ & 4 & $\geq 320$ & $\geq 512$ & 1 \\
\hline CGI0I5 & $\geq 64$ & $\geq 64$ & $\geq 64$ & 2 & 1 & 0.25 & $\leq 0.125$ & $\leq 0.25$ & $\leq 0.25$ & $\leq 1$ & $\leq 1$ & $\leq 2$ & $\leq 20$ & 32 & 0.25 \\
\hline CGI038 & $\geq 64$ & $\geq 64$ & 4 & 4 & 2 & 4 & I & $\leq 0.25$ & $\leq 0.25$ & $\leq 1$ & $\leq 1$ & $\leq 2$ & $\leq 20$ & 32 & 32 \\
\hline CGI04I & $\geq 64$ & $\geq 64$ & $\geq 64$ & 2 & I & 0.25 & $\leq 0.125$ & $\leq 0.25$ & $\leq 0.25$ & $\leq 1$ & $\leq 1$ & $\leq 2$ & $\leq 20$ & 33 & 0.5 \\
\hline CGI045 & $\geq 64$ & $\geq 64$ & $\geq 64$ & $\geq 64$ & 8 & I & 2 & $\geq 4$ & $\geq 8$ & 8 & $\geq 16$ & 16 & $\geq 320$ & $\leq 16$ & 0.5 \\
\hline CGI050 & $\geq 64$ & $\geq 64$ & $\geq 64$ & $\leq 1$ & 2 & 0.5 & $\leq 0.125$ & I & I & $\leq 1$ & $\leq 1$ & $\leq 2$ & $\leq 20$ & 32 & 16 \\
\hline CGI05I & $\geq 64$ & $\geq 64$ & $\geq 64$ & 2 & 4 & 0.25 & $\leq 0.125$ & I & I & $\leq 1$ & $\leq 1$ & $\leq 2$ & $\leq 20$ & 32 & $>64$ \\
\hline CGI070 & 2 & 16 & 4 & $\leq 1$ & 16 & 0.5 & 0.25 & $\geq 4$ & 4 & $\leq 1$ & $\leq 1$ & $\leq 2$ & $\leq 20$ & 128 & 0.125 \\
\hline CGI075 & $\geq 64$ & $\geq 64$ & $\geq 64$ & $\geq 64$ & 32 & 2 & 2 & $\geq 4$ & $\geq 8$ & $\geq 16$ & 8 & $\leq 2$ & $\geq 320$ & 64 & 0.25 \\
\hline
\end{tabular}

(Continued) 
Table 2 (Continued).

\begin{tabular}{|c|c|c|c|c|c|c|c|c|c|c|c|c|c|c|c|}
\hline \multirow[t]{2}{*}{ Isolates } & \multicolumn{15}{|c|}{ MIC $(\mu \mathrm{g} / \mathrm{mL})$} \\
\hline & ATM & CRO & CAZ & FEP & ETP & IPM & MEM & CIP & LVX & GEN & TOB & AMK & SXT & NIT & COL \\
\hline CGI079 & $\geq 64$ & $\geq 64$ & $\geq 64$ & $\leq 1$ & 4 & 0.5 & 0.5 & $\geq 4$ & 4 & 4 & 2 & 8 & $\leq 20$ & 128 & 0.125 \\
\hline CGI08I & $\geq 64$ & $\geq 64$ & $\geq 64$ & 8 & 8 & 0.25 & 0.25 & $\geq 4$ & $\geq 8$ & $\geq 16$ & $\geq 16$ & $\leq 2$ & $\geq 320$ & 32 & 0.125 \\
\hline CGI090 & $\geq 64$ & $\geq 64$ & $\geq 64$ & $\geq 64$ & 16 & I & 4 & $\geq 4$ & $\geq 8$ & $\geq 16$ & 8 & $\leq 2$ & $\geq 320$ & 64 & 0.125 \\
\hline CGI043 & $\leq 1$ & 8 & 4 & $\leq 1$ & I & I & 0.5 & $\geq 4$ & 2 & $\geq 16$ & 8 & $\leq 2$ & $\geq 320$ & 128 & 0.25 \\
\hline CGII59 & 4 & 32 & 4 & 2 & 4 & 0.25 & $\leq 0.125$ & I & 1 & 8 & 4 & $\leq 2$ & $\geq 320$ & 64 & 0.5 \\
\hline CGII8I & $\geq 64$ & $\geq 64$ & $\geq 64$ & $\leq 1$ & 2 & 1 & 0.25 & $\leq 0.25$ & $\leq 0.25$ & $\leq 1$ & $\leq 1$ & $\leq 2$ & $\leq 20$ & 32 & 0.5 \\
\hline CGI 208 & $\geq 64$ & $\geq 64$ & $\geq 64$ & 4 & 4 & 0.5 & 0.25 & 2 & 4 & 4 & 4 & $\leq 2$ & $\geq 320$ & 128 & 0.5 \\
\hline$C G|2| 2$ & $\geq 64$ & $\geq 64$ & $\geq 64$ & 2 & 2 & 0.25 & $\leq 0.125$ & I & I & $\leq 1$ & $\leq 1$ & $\leq 2$ & $\leq 20$ & 32 & 0.125 \\
\hline CGI23| & $\geq 64$ & $\geq 64$ & $\geq 64$ & 2 & 4 & 0.25 & 0.25 & $\leq 0.25$ & $\leq 0.25$ & $\leq 1$ & $\leq 1$ & $\leq 2$ & $\leq 20$ & 32 & 0.25 \\
\hline CGI236 & 32 & $\geq 64$ & $\geq 64$ & $\leq 1$ & 4 & I & 2 & $\leq 0.25$ & $\leq 0.25$ & $\leq 1$ & $\leq 1$ & $\leq 2$ & $\leq 20$ & 32 & 0.25 \\
\hline CGI244 & $\geq 64$ & $\geq 64$ & $\geq 64$ & $\leq 1$ & 2 & 0.25 & 0.5 & $\leq 0.25$ & $\leq 0.25$ & $\leq 1$ & $\leq 1$ & $\leq 2$ & $\leq 20$ & 32 & 0.125 \\
\hline CGI249 & $\geq 64$ & $\geq 64$ & $\geq 64$ & 4 & 16 & 0.5 & 4 & 0.5 & 0.5 & $\leq 1$ & $\leq 1$ & $\leq 2$ & $\leq 20$ & 32 & $>64$ \\
\hline CGI 250 & $\geq 64$ & $\geq 64$ & $\geq 64$ & 8 & 2 & 0.25 & 0.25 & 2 & 1 & $\leq 1$ & $\leq 1$ & $\leq 2$ & $\geq 320$ & 64 & 0.25 \\
\hline CGI 252 & 16 & 16 & 16 & $\leq 1$ & I & 0.5 & $\leq 0.125$ & $\leq 0.25$ & $\leq 0.25$ & $\leq 1$ & $\leq 1$ & $\leq 2$ & $\leq 20$ & 64 & 0.5 \\
\hline CGI 257 & $\leq 1$ & $\geq 64$ & $\geq 64$ & $\geq 64$ & 4 & 8 & 4 & $\leq 0.25$ & 1 & 4 & 8 & $\leq 2$ & $\geq 320$ & 64 & 0.5 \\
\hline CGI 280 & $\geq 64$ & $\geq 64$ & $\geq 64$ & 2 & I & 0.25 & $\leq 0.125$ & $\leq 0.25$ & $\leq 0.25$ & $\leq 1$ & $\leq 1$ & $\leq 2$ & $\leq 20$ & 64 & 0.125 \\
\hline CGI28I & $\geq 64$ & $\geq 64$ & $\geq 64$ & 2 & I & 0.25 & $\leq 0.125$ & $\leq 0.25$ & $\leq 0.25$ & $\leq 1$ & $\leq 1$ & $\leq 2$ & $\leq 20$ & 64 & 0.125 \\
\hline CGI330 & $\geq 64$ & $\geq 64$ & $\geq 64$ & $\geq 64$ & 16 & 4 & 4 & $\geq 4$ & $\geq 8$ & 4 & 4 & $\leq 2$ & $\geq 320$ & 64 & 0.125 \\
\hline CGI376 & 64 & $\geq 32$ & $\geq 128$ & 32 & 16 & 4 & 4 & 1 & 1 & 4 & 16 & 4 & $\geq 320$ & 64 & 0.25 \\
\hline CGI38I & $\geq 64$ & $\geq 64$ & $\geq 64$ & $\geq 64$ & 16 & 16 & 8 & 0.5 & 1 & 4 & 8 & $\leq 2$ & $\geq 320$ & 32 & 0.25 \\
\hline CGI400 & $\geq 64$ & $\geq 64$ & $\geq 64$ & 32 & 2 & I & 2 & $\geq 4$ & $\geq 8$ & $\geq 16$ & $\geq 16$ & 4 & $\geq 320$ & 64 & $>64$ \\
\hline CGI457 & $\geq 64$ & $\geq 64$ & $\geq 64$ & $\geq 64$ & 4 & 0.25 & 0.5 & $\leq 0.25$ & I & $\leq 1$ & $\leq 1$ & $\leq 2$ & $\leq 20$ & 64 & 0.5 \\
\hline CGI46I & $\geq 64$ & $\geq 64$ & $\geq 64$ & 16 & 4 & 2 & 1 & $\leq 0.25$ & 1 & $\leq 1$ & $\leq 1$ & $\leq 2$ & $\leq 20$ & 128 & 0.5 \\
\hline CGII44 & $\geq 128$ & 8 & $\geq 128$ & 4 & $\leq 0.125$ & 2 & $\leq 0.125$ & 0.5 & 0.25 & 1 & 1 & 2 & $\leq 5$ & 31 & 0.5 \\
\hline CGI479 & $\geq 64$ & $\geq 64$ & $\geq 64$ & 8 & 128 & 16 & 8 & $\leq 0.25$ & $\leq 0.25$ & $\leq 1$ & $\leq 1$ & $\leq 2$ & $\leq 20$ & 32 & $>64$ \\
\hline CGI498 & 16 & $\geq 64$ & $\geq 64$ & $\geq 64$ & 4 & 16 & I & 2 & 1 & 4 & 8 & $\leq 2$ & $\geq 320$ & 32 & 0.5 \\
\hline CGI506 & $\geq 64$ & $\geq 64$ & $\geq 64$ & 4 & 4 & 8 & 2 & I & 1 & $\geq 16$ & $\geq 16$ & 16 & $\leq 20$ & $\leq 16$ & $>64$ \\
\hline CGI522 & $\geq 64$ & $\geq 64$ & $\geq 64$ & 8 & 4 & 2 & 0.25 & $\leq 0.25$ & $\leq 0.25$ & $\leq 1$ & $\leq 1$ & $\leq 2$ & $\leq 20$ & 256 & 0.5 \\
\hline CGI532 & $\geq 64$ & $\geq 64$ & $\geq 64$ & 4 & 2 & I & 0.25 & 0.5 & 1 & 8 & 8 & $\leq 2$ & $\geq 320$ & 64 & 0.5 \\
\hline CGI547 & $\geq 64$ & $\geq 64$ & $\geq 64$ & $\geq 64$ & 16 & 16 & 16 & $\geq 4$ & 4 & $\leq 1$ & $\leq 1$ & 4 & $\geq 320$ & 128 & 0.5 \\
\hline CGI563 & $\geq 128$ & $\geq 32$ & $\geq 128$ & I & 2 & I & 0.25 & 1 & 1 & 2 & 1 & 2 & $\leq 5$ & 16 & 0.25 \\
\hline CGI565 & $\geq 64$ & $\geq 64$ & $\geq 64$ & 32 & 4 & 0.25 & 0.25 & 2 & 2 & 8 & $\geq 16$ & 32 & $\geq 320$ & 64 & 0.5 \\
\hline
\end{tabular}


Table 2 (Continued).

\begin{tabular}{|c|c|c|c|c|c|c|c|c|c|c|c|c|c|c|c|}
\hline \multirow[t]{2}{*}{ Isolates } & \multicolumn{15}{|c|}{ MIC $(\mu \mathrm{g} / \mathrm{mL})$} \\
\hline & ATM & CRO & CAZ & FEP & ETP & IPM & MEM & CIP & LVX & GEN & ТОВ & AMK & SXT & NIT & COL \\
\hline CGI574 & 2 & $\geq 64$ & $\geq 64$ & 32 & 8 & 16 & 8 & I & I & $\leq 1$ & $\leq 1$ & $\leq 2$ & $\leq 20$ & 64 & $>64$ \\
\hline CGI58I & $\geq 64$ & $\geq 64$ & $\geq 64$ & 32 & 4 & 0.5 & 0.25 & $\geq 4$ & $\geq 8$ & 8 & $\geq 16$ & 16 & $\geq 320$ & 128 & I \\
\hline CGI589 & $\geq 128$ & $\geq 32$ & $\geq 128$ & 4 & 2 & 0.5 & 0.25 & $\geq 8$ & $\geq 16$ & $\geq 128$ & $\geq 128$ & $\geq 512$ & $\geq 320$ & 128 & 0.5 \\
\hline CGI59I & $\geq 64$ & $\geq 64$ & $\geq 64$ & $\geq 64$ & 16 & 4 & 4 & $\geq 4$ & $\geq 8$ & $\leq 4$ & $\geq 16$ & $\leq 16$ & $\geq 320$ & $\leq 16$ & 0.25 \\
\hline CGI593 & 32 & $\geq 64$ & $\geq 64$ & $\geq 16$ & 2 & 8 & 2 & 0.5 & I & $\leq 1$ & 8 & $\leq 2$ & $\leq 20$ & $\leq 16$ & 0.5 \\
\hline CGI60I & 16 & $\geq 64$ & $\geq 16$ & $\leq 2$ & 1 & 2 & $\leq 0.125$ & 2 & 1 & $\geq 16$ & 8 & $\leq 2$ & $\geq 320$ & 64 & 0.5 \\
\hline CGI606 & $\geq 64$ & $\geq 64$ & $\geq 64$ & $\geq 64$ & 8 & 2 & I & $\geq 4$ & $\geq 8$ & $\geq 16$ & $\geq 16$ & $\geq 64$ & $\geq 320$ & 256 & 1 \\
\hline CGI608 & 64 & $\geq 32$ & $\geq 128$ & 16 & 16 & 16 & 8 & 1 & 4 & $\geq 128$ & 8 & 2 & $\leq 5$ & 32 & 0.5 \\
\hline CGI640 & $\geq 64$ & $\geq 64$ & 16 & 8 & $>128$ & 64 & 64 & $\geq 4$ & I & $\leq 1$ & 8 & $\leq 2$ & $\leq 20$ & 32 & 0.5 \\
\hline CGI728 & $\geq 64$ & $\geq 64$ & $\geq 64$ & 4 & 4 & 0.25 & 0.25 & $\leq 0.25$ & $\leq 0.25$ & $\leq 1$ & $\leq 1$ & $\leq 2$ & $\leq 20$ & 64 & 0.5 \\
\hline CGI737 & $\leq 1$ & $\geq 64$ & $\geq 64$ & $\geq 64$ & 64 & 8 & 8 & $\geq 4$ & $\geq 8$ & 8 & $\geq 16$ & $\leq 2$ & $\geq 320$ & 64 & 0.5 \\
\hline CGI746 & $\geq 128$ & $\geq 32$ & 64 & 32 & 4 & 0.5 & $\leq 0.125$ & $\geq 8$ & $\geq 16$ & $\geq 128$ & 32 & 4 & $\geq 320$ & 64 & 0.5 \\
\hline CGI778 & $\leq 4$ & $\geq 64$ & $\geq 64$ & $\geq 64$ & 32 & 8 & 8 & $\geq 4$ & $\geq 8$ & $\leq 1$ & $\leq 1$ & $\leq 2$ & $\geq 320$ & 64 & 0.5 \\
\hline CGI779 & $\leq 1$ & $\geq 64$ & $\geq 64$ & $\geq 64$ & 16 & 8 & 8 & $\geq 4$ & $\geq 8$ & $\leq 1$ & $\leq 1$ & $\leq 2$ & $\geq 320$ & I & 0.5 \\
\hline CGI78I & $\leq 1$ & $\geq 64$ & $\geq 64$ & $\geq 64$ & 16 & 8 & 4 & $\geq 4$ & $\geq 8$ & $\leq 1$ & $\leq 1$ & $\leq 2$ & $\geq 320$ & I & 0.5 \\
\hline CG 1813 & 8 & $\geq 64$ & $\geq 64$ & 32 & 16 & 2 & 4 & I & 2 & 8 & 8 & $\leq 2$ & $\geq 320$ & I & 0.25 \\
\hline CGI819 & $\leq 1$ & $\geq 64$ & $\geq 64$ & $\geq 64$ & 32 & 4 & 8 & $\leq 0.25$ & $\leq 0.25$ & $\leq 1$ & $\leq 1$ & $\leq 2$ & $\leq 20$ & 1 & $>64$ \\
\hline
\end{tabular}

Abbreviations: ECC, Enterobacter cloacae complex; ATM, aztreonam; CRO, ceftriaxone; CAZ, ceftazidime; IPM, imipenem; MEM, meropenem; ETP, ertapenem; CIP, ciprofloxacin; LVX, levofloxacin; GEN, gentamicin; TOB, tobramycin; AMK, amikacin; COL, colistin; NIT, nitrofurantoin; SXT, trimethoprim-sulfamethoxazole; FEP, cefepime; /, not available.

reported a significant increase in the resistance of carbapenems, making clinical treatment a great challenge. ${ }^{20,21}$ In the present study, we collected 1314 ECC clinical isolates from the First Affiliated Hospital of Wenzhou Medical University from 2004 to 2018, and 113/1314 (8.6\%) carbapenem-nonsusceptible ECC isolates were determined. The resistance of ECC strains to carbapenems showed a fluctuating upward trend from 2004 to 2018 (Table 1). In addition, the susceptibility of these 113 carbapenem-nonsusceptible ECC strains to commonly used antibacterial drugs showed that the 113 ECC strains showed a trend of multi-drug resistance as a whole. The high resistance to fluoroquinolones, cephalosporins, monobactams, and colistin, but low to amikacin, was found in our study, which corresponded to previous findings. ${ }^{22,23}$ It is worth noting that colistin resistance rate was as high as $17.7 \%$, which was related to inherent colistin resistance in the genogroups of the ECC. ${ }^{23}$ Therefore, exploring the mechanism of these strains resistant to carbapenem is important so as to better prevent resistance. As far as I know, it is first time to report the analysis of molecular mechanisms and epidemiology of carbapenemnonsusceptible ECC isolates with a longer period and large number of strains. The findings might provide a reference for the monitoring and control of carbapenemnonsusceptible ECC isolates.

The production of carbapenemase is one of the carbapenem resistance mechanisms of ECC. ${ }^{9}$ New Delhi metallo- $\beta$-lactamase (NDM), which is encoded by the gene $b l a_{\mathrm{NDM}}$, can lead to resistance to most $\beta$-lactam antibiotics, which was first found in New Delhi, India, in 2009. ${ }^{24}$ $b l a_{\mathrm{NDM}-1}$ is prevalent in the Indian region, and multiple $b l a_{\mathrm{NDM}}$ alleles have been detected in hospitals in eastern China and Czech. ${ }^{6,25,26}$ The present study also showed the 


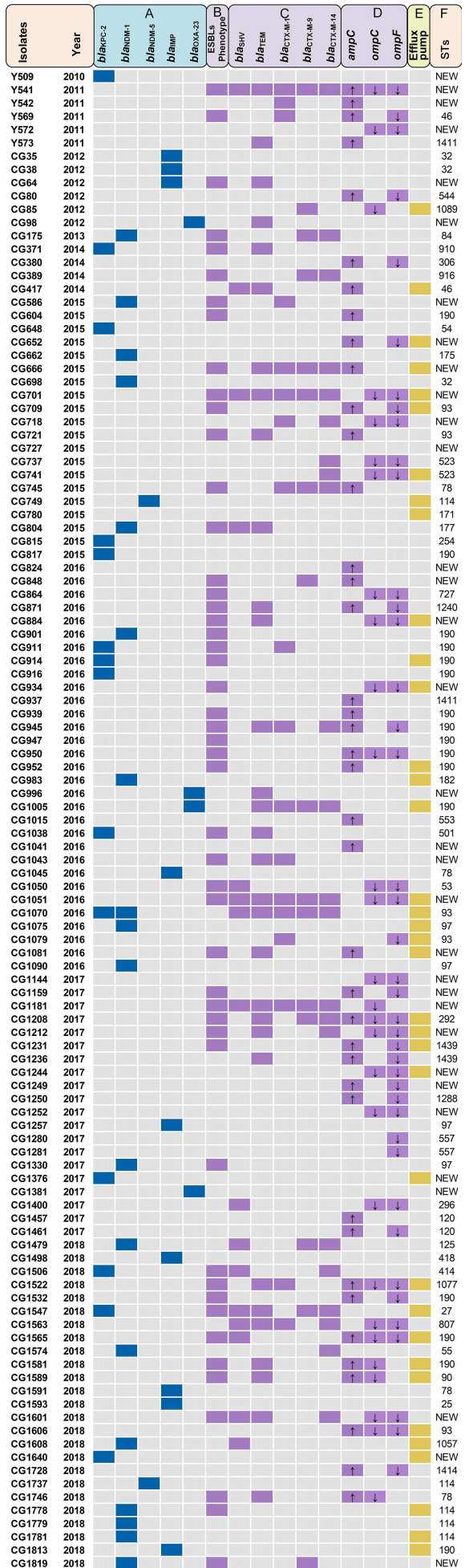

Figure I Antibiotic resistant mechanisms determined in the Enterobacter cloacae complex (ECC) isolates and multilocus sequence typing (MLST) in this study. (A) Carbapenem resistance genes; (B) extended-spectrum $\beta$-lactamases (ESBLs) phenotypic test for the phenotypic detection of ESBLs production; (C) $\beta$-lactam resistance genes; (D) expression of outer membrane porins genes and cephalosporinase gene ampC; (E) carbonyl cyanide 3-chlorophenylhydrazone (CCCP) was used to detect the activity of efflux pumps in carbapenem-nonsusceptible ECC isolates; (F) sequence typing (ST) of ECC. Blue, purple, and yellow squares represent positive. Gray squares represent negative. The purple square with an up arrow represents the overexpression of genes, and the purple square with a down arrow represents the low expression of genes. emergence and spread of NDM-1, reflecting a high prevalence since 2012. The KPC enzyme was first discovered in ECC in 2005 and is extremely endemic worldwide now. ${ }^{21,27,28}$ The dissemination of the ECC that produces $\mathrm{KPC}$ has been verified in China. ${ }^{29,30}$ In addition, a previous study reported that $8.6 \%$ KPC-2-producing strains were detected in Chongqing. ${ }^{31}$ However, our study showed that $12.4 \%(14 / 113)$ of the KPC-2-producing strains were found. VIM enzyme, which was reported in many regions, and OXA-48 enzyme, which was more common in Europe, were not found in the present study. ${ }^{32,33}$ The study also indicated an increased number of carbapenemase-producing ECC isolates over the past few years, implying that the prevalence of carbapenemnonsusceptible ECC was dependent on geographic regions and differences in drug use, even within a single country. The $b l a_{\mathrm{NDM}}$ and $b l a_{\mathrm{KPC}}$ were prevalent in carbapenemaseproducing ECC in the hospital. Therefore, effective measures need to be taken to prevent the incidence.

In addition, ESBL genes were related to increased MIC to carbapenem, which were first identified in ECC in $1989 .{ }^{34,35}$ Since then, the prevalence of ECC carrying ESBL has increased in hospital settings and in patients previously exposed to antibiotics. ${ }^{36-38}$ Compared with non-ESBL-producing strains, ESBL-producing ECC showed a higher health risk associated with hospitalacquired infections. ${ }^{39}$ In this study, 58.6\% (66/113) ECC strains produced ESBLs; most of them had more than one ESBL gene. Moreover, the ESBLs gene $b l a_{\text {TEM }}$ was popular in this study, which was totally different from the predominant bla $_{\mathrm{CTX-M-15}}$ in Bulgaria. ${ }^{40}$ We found that the carrying rates of ESBLs were higher in carbapenemresistant ECC than in carbapenem-sensitive ECC, compared with other reports. ${ }^{41}$

The production of ESBLs or the overexpression of AmpC combined with disrupted membrane permeability (outer membrane protein decreased or loss) was another reason for carbapenem resistance. ${ }^{3,9,42}$ In this study, 40 of 67 strains producing non-carbapenemase had the overexpression of AmpC or produced ESBLs combined with a decrease in the outer membrane proteins (Figure 1 and Table S2). Of the remaining 27 strains, 10 strains including 4 isolates with only decreased expression of $\operatorname{ompC}$ or/ and $o m p F, 3$ isolates with only overexpression of $\operatorname{ampC,} 2$ isolates only producing ESBLs, and 1 isolate with ESBL and overexpression of $\operatorname{ampC}$ were intermediate to carbapenem, which correspond to a previous report that the 


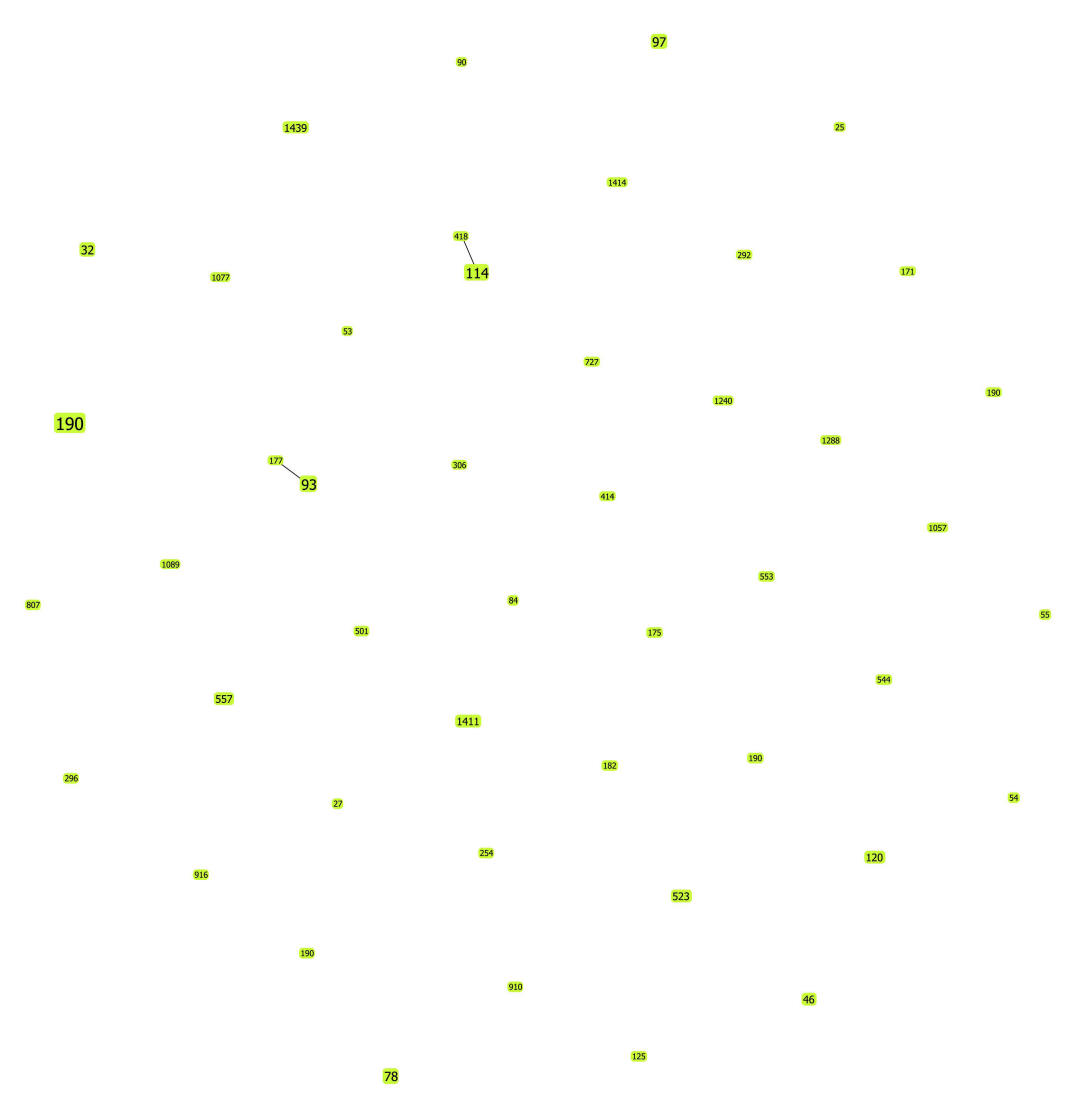

Figure 2 Performing goeBURST analysis on the molecular epidemiological characteristics of $79 \mathrm{ECC}$ isolates. The population snapshot indicates the clonal assignment of the sequence typing (ST) presented in this study. Each green dot represents one ST, the numbers in the dot represent ST types, and the dot size represents their abundance in the ST set.

overexpression of $\operatorname{ampC}$ genes encoding cephalosporinase, leading to ECC strains resistant to first- and secondgeneration cephalosporins, and the disrupted membrane permeability (outer membrane protein decreased or loss) slightly increased MIC to carbapenem but did not lead to resistance. ${ }^{35}$ However, 15 other ertapenem-resistant strains were also found in the aforementioned mechanism combinations. We suspected that these combinations might increase the MIC of these strains to carbapenem drugs and even reach the level of resistance (Table S2), which still requires further exploration.

Several studies reported the effect of the efflux pump on the carbapenem resistance of ertapenem-insensitive ECC strains. ${ }^{9,42}$ The present study indicated that CCCP, an efflux pump inhibitor, decreased the MIC of 36 ECC strains to carbapenem, suggesting that efflux pumps played an important role in carbapenem resistance. However, the expression of the efflux pump acrB revealed no difference in ertapenem-resistant ECC strains, suggesting the presence of an additional unknown efflux pump influencing ertapenem resistance. ${ }^{9}$
The results of MLST analysis suggested that the distribution of STs of all carbapenem-nonsusceptible ECC isolates was diversified (Figure 2). ST190 was the most prevalent isolate in the hospital; a small-scale explosion in 2016 (Figures 1 and 2), different from previous studies, revealed that ST66, ST78, ST108, and ST114 were the most prevalent and widespread ECC STs. ${ }^{35,43}$ The present study found that ST190 isolates producing carbapenemase and various other $\beta$-lactamase profiles with a higher risk might cause severe drug-resistant outbreaks in the hospital. ST78 and ST114 producing carbapenemase were major international clones, which were worth noting and reminded us of the spread of these strains.

In conclusion, this study summarized the resistance mechanisms and molecular epidemiology of carbapenemnonsusceptible ECC strains in the hospital from 2004 to 2018. This was the first time that ECC nonsusceptible to carbapenem antibiotics was reported on a large scale in China. The increasing rates of resistance to antibiotics have further aggravated the threat to human health because of limited treatment options. ECC isolates that 
do not produce carbapenemase are predominant in carbapenem-nonsusceptible ECC isolates. Carbapenem resistance is mediated by the overexpression of efflux pumps, or, more commonly, through the acquisition of constitutive overexpression of AmpC or ESBL combined with a decrease in the outer membrane proteins. The resistance of carbapenemase-producing ECC isolates is conferred through the acquisition of carbapenemase genes and the overexpression of efflux pumps. As carbapenem antibiotics are gradually applied as an effective treatment option, monitoring programs to prevent the emergence and further spread of antibiotic resistance are urgently needed.

\section{Ethics Approval}

The need for ethics approval and consent was deemed unnecessary in this study according to the ethics committee of the First Affiliated Hospital of Wenzhou Medical University.

\section{Acknowledgments}

We thank the Health Department of Zhejiang Province of the People's Republic of China (no. 2011KYA106) for providing financial funding.

\section{Author Contributions}

All authors made a significant contribution to the work reported, whether that is in the conception, study design, execution, acquisition of data, analysis and interpretation, or in all these areas; took part in drafting, revising, or critically reviewing the manuscript; gave final approval of the version to be published; have agreed on the journal to which the manuscript has been submitted; and agree to be accountable for all aspects of the work.

\section{Disclosure}

The authors declare no conflicts of interest.

\section{References}

1. Sanders WE Jr., Sanders CC. Enterobacter spp.: pathogens poised to flourish at the turn of the century. Clin Microbiol Rev. 1997;10:220-241. doi:10.1128/CMR.10.2.220-241.1997

2. Davin-Regli A, Pages JM. Enterobacter aerogenes and Enterobacter cloacae; versatile bacterial pathogens confronting antibiotic treatment. Front Microbiol. 2015;6:392. doi:10.3389/fmicb.2015.00392

3. Annavajhala MK, Gomez-Simmonds A, Uhlemann AC. Multidrugresistant Enterobacter cloacae complex emerging as a global, diversifying threat. Front Microbiol. 2019;10:44. doi:10.3389/ fmicb.2019.00044
4. Huang S, Dai W, Sun S, et al. Prevalence of plasmid-mediated quinolone resistance and aminoglycoside resistance determinants among carbapenem non-susceptible Enterobacter cloacae. PLoS One. 2012;7(10):e47636. doi:10.1371/journal.pone.0047636

5. Corkill JE, Anson JJ, Hart CA. High prevalence of the plasmid-mediated quinolone resistance determinant qnrA in multidrug-resistant Enterobacteriaceae from blood cultures in Liverpool, UK. J Antimicrob Chemother. 2005;56(6):1115-1117. doi:10.1093/jac/dki388

6. Jin C, Zhang J, Wang Q, et al. Molecular characterization of carbapenem-resistant Enterobacter cloacae in 11 Chinese cities. Front Microbiol. 2018;9:1597. doi:10.3389/fmicb.2018.01597

7. Raimondi A, Traverso A, Nikaido H. Imipenem- and meropenem-resistant mutants of Enterobacter cloacae and proteus rettgeri lack porins. Antimicrob Agents Chemother. 1991;35 (6):1174-1180. doi:10.1128/aac.35.6.1174

8. Galani I, Souli M, Chryssouli Z, et al. Characterization of a new integron containing bla(VIM-1) and aac(6')-IIc in an Enterobacter cloacae clinical isolate from Greece. J Antimicrob Chemother. 2005;55(5):634-638. doi:10.1093/jac/dki073

9. Yang FC, Yan JJ, Hung KH, et al. Characterization of ertapenem-resistant Enterobacter cloacae in a Taiwanese university hospital. J Clin Microbiol. 2012;50(2):223-226. doi:10.1128/ JCM.01263-11

10. Boyd DA, Mataseje LF, Davidson R, et al. Enterobacter cloacae complex isolates harboring bla NMC-A or bla IMI-type class a carbapenemase genes on novel chromosomal integrative elements and plasmids. Antimicrob Agents Chemother. 2017;61(5). doi:10.1128/AAC.02578-16

11. Van Maerken T, De Brabandere E, Noel A, et al. A recurrent and transesophageal echocardiography-associated outbreak of extended-spectrum beta-lactamase-producing Enterobacter cloacae complex in cardiac surgery patients. Antimicrob Resist Infect Control. 2019;8(1):152. doi:10.1186/s13756-019-0605-4

12. Arpin C, Labia R, Dubois V, et al. TEM-80, a novel inhibitorresistant $\beta$-lactamase in a clinical isolate of Enterobacter cloacae. Antimicrob Agents Chemother. 2002;46(5):1183-1189. doi:10.1128/ aac.46.5.1183-1189.2002

13. Bornet C, Davin-Regli A, Bosi C, et al. Imipenem resistance of Enterobacter aerogenes mediated by outer membrane permeability. $J \quad$ Clin Microbiol. 2000;38(3):1048-1052. doi:10.1128/ JCM.38.3.1048-1052.2000

14. Pitout JD, Moland ES, Sanders CC, et al. Beta-lactamases and detection of beta-lactam resistance in Enterobacter spp. Antimicrob Agents Chemother. 1997;41(1):35-39. doi:10.1128/AAC.41.1.35

15. Szabo D, Melan MA, Hujer AM, et al. Molecular analysis of the simultaneous production of two SHV-type extended-spectrum beta-lactamases in a clinical isolate of Enterobacter cloacae by using single-nucleotide polymorphism genotyping. Antimicrob Agents Chemother. 2005;49(11):4716-4720. doi:10.1128/ AAC.49.11.4716-4720.2005

16. Chavda KD, Chen L, Fouts DE, et al. Comprehensive genome analysis of carbapenemase-producing Enterobacter spp.: new insights into phylogeny, population structure, and resistance mechanisms. mBio. 2016;7(6):e02093-e02116. doi:10.1128/mBio.02093-16

17. Fernandes CJ, O'Sullivan MV, Cai Y, et al. Agar dilution method for detection of inducible clindamycin resistance in Staphylococcus spp. J Clin Microbiol. 2007;45(12):4018-4020. doi:10.1128/JCM.01158-07

18. Silago V, Kovacs D, Samson H, et al. Existence of multiple ESBL genes among phenotypically confirmed ESBL producing Klebsiella pneumoniae and Escherichia coli concurrently isolated from clinical, colonization and contamination samples from neonatal units at Bugando Medical Center, Mwanza, Tanzania. Antibiotics. 2021;10:476. doi:10.3390/antibiotics10050476 
19. Nicolau DP, Carmeli Y, Crank CW, et al. Carbapenem stewardship: does ertapenem affect Pseudomonas susceptibility to other carbapenems? A review of the evidence. Int J Antimicrob Agents. 2012;39 (1):11-15. doi:10.1016/j.ijantimicag.2011.08.018

20. Gupta V, Ye G, Olesky M, et al. National prevalence estimates for resistant Enterobacteriaceae and Acinetobacter species in hospitalized patients in the United States. Int J Infect Dis. 2019;85:203-211. doi:10.1016/j.ijid.2019.06.017

21. Wilson BM, El Chakhtoura NG, Patel S, et al. Carbapenem-resistant Enterobacter cloacae in Patients from the US veterans health administration, 2006-2015. Emerg Infect Dis. 2017;23(5):878-880. doi:10.3201/eid2305.162034

22. Pot M, Reynaud Y, Couvin D, et al. Wide distribution and specific resistance pattern to third-generation cephalosporins of Enterobacter cloacae complex members in humans and in the environment in Guadeloupe (French West Indies). Front Microbiol. 2021;12:628058. doi:10.3389/fmicb.2021.628058

23. Mushtaq S, Reynolds R, Gilmore MC, et al. Inherent colistin resistance in genogroups of the Enterobacter cloacae complex: epidemiological, genetic and biochemical analysis from the BSAC Resistance Surveillance Programme. $J$ Antimicrob Chemother. 2020;75 (9):2452-2461. doi:10.1093/jac/dkaa201

24. Yong D, Toleman MA, Giske CG, et al. Characterization of a new metallo- $\beta$-lactamase gene, bla NDM-1, and a novel erythromycin esterase gene carried on a unique genetic structure in Klebsiella pneumoniae sequence type 14 from India. Antimicrob Agents Chemother. 2009;53(12):5046-5054. doi:10.1128/AAC.00774-09

25. Wang Q, Wang X, Wang J, et al. Phenotypic and genotypic characterization of carbapenem-resistant Enterobacteriaceae: data from a longitudinal large-scale CRE study in China (2012-2016). Clin Infect Dis. 2018;67(suppl_2):S196-S205. doi:10.1093/cid/ciy660

26. Paskova V, Medvecky M, Skalova A, et al. Characterization of NDM-encoding plasmids from Enterobacteriaceae recovered from Czech hospitals. Front Microbiol. 2018;9:1549. doi:10.3389/ fmicb.2018.01549

27. Park SO, Liu J, Furuya EY, et al. Carbapenem-resistant Klebsiella pneumoniae infection in Three New York city hospitals trended downwards from 2006 to 2014. Open Forum Infect Dis. 2016;3(4): ofw222. doi:10.1093/ofid/ofw222

28. Bratu S, Mooty M, Nichani S, et al. Emergence of KPC-possessing Klebsiella pneumoniae in Brooklyn, New York: epidemiology and recommendations for detection. Antimicrob Agents Chemother. 2005;49(7):3018-3020. doi:10.1128/AAC.49.7.3018-3020.2005

29. Zhao Y, Zhang J, Fu Y, et al. Molecular characterization of metallo-betalactamase- producing carbapenem-resistant Enterobacter cloacae complex isolated in Heilongjiang Province of China. BMC Infect Dis. 2020;20(1):94. doi:10.1186/s12879-020-4768-7

30. Huang J, Ding H, Shi Y, et al. Further spread of a blaKPC-harboring untypeable plasmid in Enterobacteriaceae in China. Front Microbiol. 2018;9:1938. doi:10.3389/fmicb.2018.01938

31. Dai W, Sun S, Yang P, et al. Characterization of carbapenemases, extended spectrum beta-lactamases and molecular epidemiology of carbapenem-non-susceptible Enterobacter cloacae in a Chinese hospital in Chongqing. Infect Genet Evol. 2013;14:1-7. doi:10.1016/j. meegid.2012.10.010

Infection and Drug Resistance

\section{Publish your work in this journal}

Infection and Drug Resistance is an international, peer-reviewed openaccess journal that focuses on the optimal treatment of infection (bacterial, fungal and viral) and the development and institution of preventive strategies to minimize the development and spread of resistance. The journal is specifically concerned with the epidemiology of
32. Albiger B, Glasner C, Struelens MJ, et al. Carbapenemase-producing Enterobacteriaceae in Europe: assessment by national experts from 38 countries, May 2015. Euro Surveill. 2015;20(45):30062. doi:10.2807/1560-7917.ES.2015.20.45.30062

33. Bitar I, Papagiannitsis CC, Kraftova L, et al. Detection of five mcr9 -carrying Enterobacterales isolates in four Czech hospitals. mSphere. 2020;5(6):e01008-e01020. doi:10.1128/mSphere.01008-20

34. De Champs C, Sauvant MP, Chanal C, et al. Prospective survey of colonization and infection caused by expanded-spectrum-betalactamase-producing members of the family Enterobacteriaceae in an intensive care unit. J Clin Microbiol. 1989;27(12):2887-2890. doi:10.1128/JCM.27.12.2887-2890.1989

35. Cai Y, Chen C, Zhao M, et al. High prevalence of metallo-betalactamase-producing Enterobacter cloacae from three tertiary hospitals in China. Front Microbiol. 2019;10:1610. doi:10.3389/ fmicb.2019.01610

36. Kluytmans-van den Bergh MF, Rossen JW, Bruijning-Verhagen PC, et al. Whole-genome multilocus sequence typing of extended-spectrumbeta-lactamase-producing Enterobacteriaceae. $J$ Clin Microbiol. 2016;54(12):2919-2927. doi:10.1128/JCM.01648-16

37. Jean SS, Hsueh PR, Group SA-P. Distribution of ESBLs, AmpC betalactamases and carbapenemases among Enterobacteriaceae isolates causing intra-abdominal and urinary tract infections in the AsiaPacific region during 2008-14: results from the Study for Monitoring Antimicrobial Resistance Trends (SMART). J Antimicrob Chemother. 2017;72:166-171. doi:10.1093/jac/dkw398

38. Peirano G, Matsumura Y, Adams MD, et al. Genomic epidemiology of global carbapenemase-producing Enterobacter spp., 2008-2014. Emerg Infect Dis. 2018;24(6):1010-1019. doi:10.3201/eid2406.171648

39. Cyoia PS, Koga VL, Nishio EK, et al. Distribution of ExPEC Virulence Factors, bla CTX-M, fosA3, and mcr-1 in Escherichia coli isolated from commercialized chicken carcasses. Front Microbiol. 2018;9:3254. doi:10.3389/fmicb.2018.03254

40. Markovska R, Stoeva T, Dimitrova D, et al. Quinolone resistance mechanisms among third-generation cephalosporin resistant isolates of Enterobacter spp. in a Bulgarian university hospital. Infect Drug Resist. 2019;12:1445-1455. doi:10.2147/IDR.S204199

41. Hoffmann H, Sturenburg E, Heesemann J, et al. Prevalence of extended-spectrum beta-lactamases in isolates of the Enterobacter cloacae complex from German hospitals. Clin Microbiol Infect. 2006;12(4):322-330. doi:10.1111/j.1469-0691.2006.01360.x

42. Szabo D, Silveira F, Hujer AM, et al. Outer membrane protein changes and efflux pump expression together may confer resistance to ertapenem in Enterobacter cloacae. Antimicrob Agents Chemother. 2006;50(8):2833-2835. doi:10.1128/AAC.01591-05

43. Izdebski R, Baraniak A, Herda M, et al. MLST reveals potentially high-risk international clones of Enterobacter cloacae. J Antimicrob Chemother. 2015;70(1):48-56. doi:10.1093/jac/dku359

\section{Dovepress}

antibiotic resistance and the mechanisms of resistance development and diffusion in both hospitals and the community. The manuscript management system is completely online and includes a very quick and fair peerreview system, which is all easy to use. Visit http://www.dovepress.com/ testimonials.php to read real quotes from published authors. 